\title{
DE M. CORNELIO FRONTONE
}

\author{
FIUSQUE SCRIPTIS.
}

M. Connerius Fronto, (1) natus est Cirtæ (2) in Numidia, quod oppidum non solum iure coloniæ florebat, sed, collocatis in ea sede Sittianis militibus, Divo Iulio dictatore, ab Italis habitari coprum est (3). Itaque quum se ipse Afrum dicit, Afrumque Numidam (4), non id adeo premendum est ut enm inde efficids pere rrina stirpo ortum alio quam latino sermone patrio usum esse; quamquam id quidem certissimum est, quum in tam $m$ 'gnam tamque populosam civitatem deducerentur coloni illi militares, non ideo pulsos esse habitatores veteres, sed sensim cum is qui initio deductæ coloniz soli cives essent, coaluisse (5). Atque fuisse inter colonias provinciæ Africe, apud quas latina lingua in usu non

(x) Plena illa tria nomina Pisaurensis exhibet inscriptio, quum scriptores apud quos eius meitio extat plerique, omisso praenomine, modo Cornelium Frontonem, mudo pro etus aevi mort Frontonem Cornelium appellent; ali, cum Codice Mediolanensi, II. Frontonem.

(.2 Cirtensem dicit Minutius Felix (cap. 9. collato cum cap. 3r.), municipem Cirtensem se ipse Fronto fert manifeste (Epp. ad Amicus II, 6;.

(3) De Cirta vid. quae diximus ad E.p. modo laudatam.

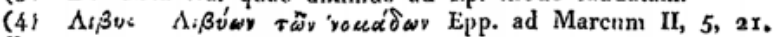
Gallorum quorundam opinionem, qui ex male inteliecto $S$ udonii loco originem ei liquitanican aftinxerant, iam dudum Bay us refutarit v. Fronton.

(5) Ne ipsa qurlasn Inlia Corinthus, in dirutae urbis solo locata, adscitis ex viculad Graecis, adeo din sermonis latini et morum, praeter gladiatorium furorem, tenax fuit. 
esset, vel ex Appuleio compertum est: scimus enim honoris causa plurima in provinciis oppida, incolis non mutatis, coloniarum titulo insignita esse. Frontonem autem ab Itało genere originem duxisse vel ex ipso Cornelii nomine coniicere licet: nota enim sunt illa tot millia Corneliorum, qui a L. Sulla Dictatore civitatem accepernnt: ex quibus plurimos, dissipatis luxu divitiis per arma male quæsitis, cum nihil in duce præter fortunam audaciamque spectarent, P. Sittio exulibusque et Sertorianis quos ille ductbat, sese iunxisse, qui illorum temporum exempla reputaverit pro certissimo habebit. Natum esse Frontonem imperante Domitiano Aug. inde patet quod iam sub Hadriano tam clarus evaserat, præ. sertim quum appareat serum eum accessisse ad studia: quippe qui, duo et viginti annos natus, vix quidquam veterum lectionum attigerat (1). In epistolis quas primis Divorum Fratrum scripsit annis, senem se, fessumque vitæ exhibet (2).

Alexandriæ quum plurimos se familiares habuisse dicat (3), ibi eum aliquamdiu commoratum esse, studiorum fortasse causa, coniectes: et commeabatur ea æatate frequenter inter Alexandriam provinciamque Africam terrestri itinere, ubi nunc horridæ sunt solitudines. Athenis vero et in Græcia Frontonem litteris studuisse haud crediderim: non tantum quod nullum huius rei in eius scriptis vestigium reperitur, sed quod sui evi Græcos rhetoras vehementer despicit (4): quod non fecisset qui in illorum scholis adolevisset. Habuit quidem Græcum magistrum, Dionysium tòv $\lambda_{\xi} \pi \tau \dot{0}$, quem ipse, antiqui latini sermonis in huiusmodi cognomini-

(x) Epp. ad M. Caesarem II. 4, xo.

(2) Epp. ad Amicos I. I, 5. De nepote amisso ep. 2. 5.

(3) Ad T. Pium Imp. ep. S, 4.

(4) Polemonem certe, qui sub Divo Pio graecorum rhetorum princeps habebatur: Epp. ad Marcunı U. 3. ibid. 4, ro. Neqne de Herode eun marnitice seusisse, satis apparet; - ad L. Verum ep. 8. Arque id quidem nos vehementer probanus; sed fieri potest, ut qui contemptum mereatur, ab homiue contemnatur nequaquam praestantiore. 
bus morem secutus, Tonuioren dicit (1): sed Latinos suos præ Gracis miratur; ut de ipso, quod Cicero de L. Cælio, dicere possis, argutias illum suas ex librariolis Latinis hausisse.

Romam postquam se contulerat, famam magnam, iam sub Divo Hadriano, adeptus est inter causidicos(2), oratoriaque artis magistros. Itaque ei hac disciplina instruendus traditus est puer ut videtur (3), M. Annius Verus, qui postea M. Antonini nomine Imperium obtinuit: serius L. Commodus, cui Veri Aug. nomen obtigit. Hinc Frontoni honorifica Magistri Augg. appellatio in Pisanxensi insoriptione tribuitur, eademque uterque Augustus litteras ad illum datas inscribere consueverat. Multos preterea alios instituit nobiles iuvenes: quos domi et in contubernio suo secum habebat (4). Itaque ut quastuosissinum illis temporibus erat et rhetoris munus et causidici, amplas consecutus est divitias, ita ut Mæcenatianos hortos possidoret (5), et balneas ædificaret, quarum sumptus emplius trecentis sestertiis constaret (6). Nam quod se pauperem dicit, causa hæc est, quod opes vel maximæ munificentia Principum amicarum collatæe, industriaque quæsitæ ab homine non avaro, zenues erant ubi cum immensis Romanorum nobilium divitiis comparabantur.

In Senatum iam ab Hadriano Imp. lectum fuisse verisimile est: id vero certissimum, quamquana omissa vetere honorum successione, non nisi qui senator esset consulem factum : et mansisse in Sematu qui consul fuis-

(r) Ad M. Caesarem de Eloq. fr. ro.

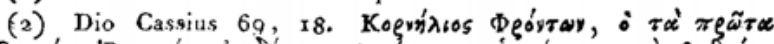

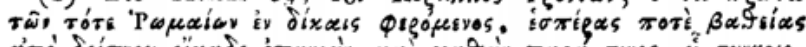

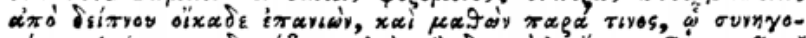

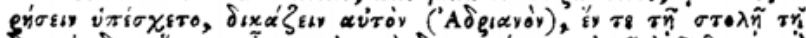

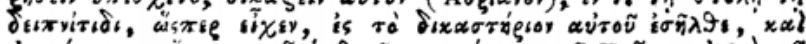

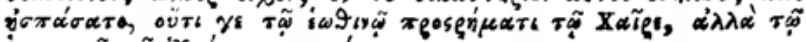

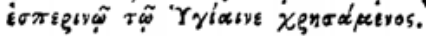

(5) Ad M. Caesarem de Eloq. fr. 9, 5.

(4) Epp. ad Amicos I. 13, 14.

(5) Epp. ad M. Caesarem II. 4, 13.

(6) A. Gellius XIX. c. 10. 
set. Senatores idea sui ordinis viros, se Senatorem vo. cat Fronto (1). Consulatum gessit bimestrem (2), mensibus Iulio et Augusto (3), cum Marcus Cæsar duos et viginti anuos natus esset (4), quod, ut recte vidit Maius, incidit in 4. 243 , Coss. ordinariis T. Bellicio Torquato, Ti. Claudio Herode Attico. Sortitus deinde regendam proconsularem provinciam, vel Achaiam, vel aliquim ex Asianis, T. Pio Imp. sese excusavit (5) propter valetudinem, quæe etsi ei ita gravis obtigit ut vera esse causa potuerit, tamen nescio an sumptus causa, qui ea ctate permagnus fuit, proconsulatum detrectandum censuerit. Nam cum olim provinciis diripiendis proconsules Romani divitias collegissent, tuvc is honor erat qui patrimonium consumeret, adeo ut principum munificentia onera amicorum susciperet, que proconsulatu fungi possent (6). Patriæ negotia patronus Romæ gessit, donec senio et valetudine fatigatus eo se munere abdicsret (7)

Articulorum pedumque doloribus graviter conflictatus, et prope ad vitie tredium adactus est (8). Itaque quim sæpissime domi ægrotus decumberet, conveniebant ad eum plurimi qui tius sermonibus purissimis, bonaramque doctrinarum plenis delectarentur (9). Et

(1) E.pp ad Amieos I. 4, 8. Fpp. ad Verum Aug. 6, 2.

(2) Epp. ad $\mathrm{M}$. Causarem II. 6, 9. Id quidem iam notum erat ex. Ausouio in gratiarum actione.

(i) E.pp. ad M. Caesarem II. 7 .

(4) Epp. ad M. Caesarem II. 4, 10.

(5) Epp. ad T. Pium Aug 8.

(b) Iul. Capitolinus in Marco, c. 2. Proculum usque ad proconsulatum provexit, oueribus iu se receptss.

(7) V. adnot. nostr. x. p. 2 I5.

(8 Plenae sunt hae episfolae querelarum Frontonis, commiserationisque Marci. Vide etiam Gellium II. c. 26. XIX. c. ro. Qua* de arthritide Frontonis narrat Artemidoris IV. c. 24.

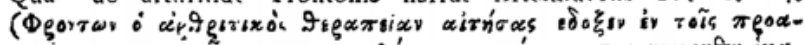

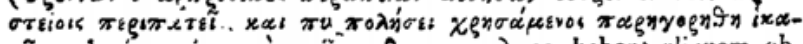

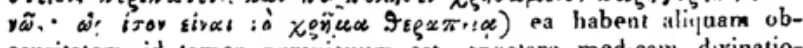
scuritatem, id tamen perspicuum est, spectare medicam divinationis vim, quae illa aetdie, ut evemplis 21 . Antonini et Aelii Aristidis iscemur, in sin. its max.me efticacem praestitit.

(9) Sunt haec rerba Geili XLX, 8. 
magno honore vivum apud cortaneos habitum esse tum ex Gellio constabat, tum reliquia nostræ confirmant: insecuta xtas ut perfecti oratoris exemplum ita admirata est ut Oratoris nomen nancisceretur tanquam proprium (1), neque multum aberat quin Ciceroni par censeretur (2). Laudant gravitatem Frontonis (3): eidem quum siccum genus dicendi gravitati contrarium tribuunt (4), laudem laude cunulant.

Quin nata in eius disciplina est mansitque secta Frontonianorum (5), qui, quantum coniectura assequi licet, a ceteris oratorum familiis ex sophistarum Græcorum institutione profectis eatenus diversi fuisse videntur, quod tumidum floridumque orationis genus vitarent, ac de verborum delectu, maxime ex priscis scriptoribus Romanis, solliciti essent: quæ cura, cum accurata sermonis patrii cognitione coniuncta, laudem illam eximiam Frontoni nostro videtur comparasse: quum alii eius rtatis scriptores nulla severitate et pravis vocabulis uterentur, et in

(x) Ita in inscripr, Pisaur. (p. x45. ed, n.)

(2) Eumenius cap. 14. Fronto Romance eloquentice non secundum sed alterum decus. Ausonits (irat. Act. In tanti te oratoris fastigium attollis? Eutropins VIII. - Fronto orator nobilissimus. Prudentius fecit Martianus Capella 1. V. p. 138. qui Frontonem cum Regulo Plinioque inter minores oratores numerat. (Moneo me hilec et similia de Froutons testimonia debere Maio qui ea collegit)

(3) S. Hieronymus Lp. ad Rusticum c. r2. Sidonius Apollin. IV, 8 .

(4) Macrobins: Saturn. V. $x$

(5) Sidonius Apoll. I. x. Unde paret, Iulium Titianum fuisse de Trontonianis, eo dirersum a consectaneis, ut Sidonius eos appellat, quod ille initaretur, quod ceteri ut veternosum contemuerent, dicendi genus Tullianum. Ipse Sidonius non alienus censendus est ab hac secta: nam quod simul cum illa orationis forma, quae sibi placeret, contrariam adsciscebat, id faciebat pro seculi ınore quem ex philusophis cognoscimns. Hit cernere milhi visus sum in Sidonio vestigia initationis Frontonianae, et Sidoninm Panegyricosque cum nostro oratore comparandos esse moneo. Liceat hic obiter observare, sententiam illam non nominati oratoris, quam Sidonius adsumit VII. x4. Filium MI. Ciceronis pop. Rom. non agnuscelat loquentem, petitain videri ex Frontone: quod mihi persuadet conformatio numeri qui idem plane est atque in illa sententia Ornibus - insignis: Epp. ad M. Caes. II. 4, $x$. 


\section{XXIV}

tumorem effrisi poetarum verba adsciscerent, neo quid. quam pro diserte dicto haberent quod simplex verum. que esset.

Sed a Frontonis oratione sensus validos sanosque abesse, qui soli sine fuco placere possunt, seculi perversitats non intellectum est

Marçum adolescentia egressum ab oratoria arte desoivisse, ut ex ipsius commentariis notum erat, ita Frontonis epistola, quam de eloquentia inscripsi, plenius docemur. Is tamen ipse, ex quo ad Imperium pervenerat, quod in Senatu cum oura verba facere debebat, ad veteris magistri disciplinam reversus est. Honorificentius etiam de mayistro sensit L. Verns, qui eum, ut historiam belli Parthici scriberet impense rogans, fore arbitrabatur ut gesta suo porteris laita viderentur quartta ille narratione sua 'a videri vellet (1'.

Quanta caritate Marcus, et iuvenis Frontonem dilexerit et per omnem atatem coluexit, ex utriusque litteris iam patet. Plus eum Frontoni quam ceteris quibus nsus est rhetoricis et grammaticis magistris tribuisse, Capitolinus diserte docet ( 2 ): neque, opinor, ille æmulum tulisset. Stgtuam Frontoni Marcum a Senatu petiisse, idem Capirolinus testis est (3): mortuo creda; quod de Rustico certo constat(4). Nec dubium, quum Marcus ,gtantiom honoris magistris suis detulerit ut imagines „,eorum aureas in latario habevet, ac sepulcra eorum „aditu, hostijs, fluribus, semper honararet“ (5), quin Frontonis memoriam eadem pietate coluerit.

Șed hunc Marci amorem non tam vana præclaræ eloquentia opinione quam vera virtutis laude, candidoque anima et Q.joorrgria illa quam in Romanis desiderat Fronto noster (6), conciliatum fuisse, vel inde patet, quod commentariorum initio, ubi gratam commemofationen facit beneficiorum a singulis in se collatorum, de eloquentia quidem Frontonis nihil quidquam profert,

(x) Epp. ad Ferum Aug. 7, 15 . (a) Iul. Gapitol. in Marro c. 2 (5) Ibid. (4) Idem ibid. c. 3. (5) lbid. (b) Ad $I$. Fefum $6, x x_{4}$ 
sed hoc se ab illo accepisse predicat, quod didicerit, ,quæ esset tyrannorum invidia et simulatio, et Roma"nos nobiles sincero caritatis amicitiæque sensu desti"tui" (1). Ac profecto virum bonum fuisse Frontonem, et qualem tibi amicum exoptes, ex reliquiis, quæ in luçem prolatæ sunt, iam efficitur. In Christianos quidera iniquissimus fuit, quos falsis infandorum scelerum criminibus oneravit; sed nemini vitio vertas, $s i$ in talibus rebus partium studio decipiatur.

De patre Nostri et genere nilhil notum: matrem a Plutarcho Chéeronensi genus duxisse, recentioris retatis commentum est (2). Fratris ipse meminit, cum quo concordissime vixit; eum Divi Pii bonitate summos honores adeptum esse dicit, quod de consulatu intelligendum videtur (3). Uxorem, seu Gratiam sive Cratiam vocatam (4), sibi per multos annos caram, senex amisit antequam L. Verus ex Asia rediret (5).

Filiam unicam Aufidio Victorino collocavit (6), summo belli pacisque artibus viro (7). Ea plures illi nepotes peperit, quorum unum avus domi sure educabat: is trimulus defunctus est bello Parthico aüfecto (8). Alterum avus nunquam vidit, natum mortunmque in Germąnia ubi Aufidius exercitum adversos Cattos ducebat (9). Tertius nepos erat M. Aufidius Fronto, Consul a. 199. qui Pisaurense monumentum filio posuit infanti; cuius fratrem fuisse C. Aufidium Victorinum, anni 200. Consulem, merito suspicatur Oliverius. Igitur cum Sidonius Apallinaris ad amicum Leonem, qui multum posset in aula Gothica Tolosæ, scribat (10): Perorandi illud flumen quod non solum gentilitium sed domesticum tibi, quodque in tuum pectus per succiduas aetates ab atavo Frontone

(r) Comment, I. c. $\mathbf{x}$.

(2) Auctorem citant Iohannem Sarisberiensem, sed parum attente lectum: nam Sextum cum Frontone confundunt.

(3) De nepote amisso 2, 24. (4) Ad M. Caesarem II, 8. 9.

(5) Ad L. Verum 8, 1. 9, 2.

(6) De nepote amisso, passim, Ad Amicos I, r. I, 6, 3. Inscr. Pisaur.

(7) p. x 47. adn. r. (8) Ad L. Verum 8, x. 9, 2.

(9) v. Libellus de nep. amisso, (ro) Epp. VIII. 3. 


\section{XXVI}

transfunditur: is Leo ex posteris Aufidii Viotorini fuerit necesse est. Atavum a Sidonio Frontonem dici ut generis auctorem, non propria vocabuli significatione, tempora reputanti perspicuum est: eodem modo quo Auctor Crat. de Harusp. resp. Appium Cæcum P. Clodii atavum riscat.

Nulla Frontonis extat epistola recentior illa quam ad Verum ex Asia reducem scripsit (1). Grandævum virum senio paulo post defunctum esse, verisimile est: nisi peste ablatus sit qua illo tempore orbem Romanum devastavit.

\section{Scripta Frontonis.}

Ex omnibus Frontonis libris ante A. Maium unus de differentiis vocabulorum ad nostram ratem pervenisse videbatur: misere corruptus ille, ut fere omnes minores grammatici latini, et ita vilis nugarumque plenus, ut probabile visum haud fuerit tale quidquam a Frontonis ingenio potuisse proficisci. Neque iis qui iam in lncem prodierunt, argumenta ad hanc controversiam dirimendam continentur, nisi quod, perspecto Frontonis ingenio, vetamur ei abiudicare librum quod minimi sit pretii. De editionibus huius libri, earumque familiis et indole, ad eins initium disseruit Buttmannus.

Itaque illa qua Maius ex Codice Ambrosiano edidit, incognita inauditaque erant omnia præter paucissimos versus quos inde Charisius citaverat. Sed cum longe frequentissima apud veteres Frontonis oratoris mentio sit, non oratorem ea illum nobis exhibent, sed epistolographum : paucissimis exceptis paginis que ipsæ epistolurum libris insertie erant. Atque omnia sedulo consideranti mihi videntur Frontonis scripta olim in duo corpora digesta fuisse, orationum alterum, alterum epistolarum, quorum illud alio plane volumine scriptum

(t) Ad L. Verum Aug, re. 
fuerit $a b$ eo diverso, cuius partes Bobii in usum rescribendi cullatæ sunt.

Sequitur iam ut indicem exhibeamus redivivorum illorum librorum. Monendi vero lectores sunt, cum librorum titulos recensitos legant, vix unum aut alterum ex iis esse integrum; plerosque magna, quin maxima parte nutilos extare.

1. L:ber epistolarum ad T. Antoninum Pium Ang. quæ quibus annis scriptæ sint, accuratius definiri nequit. Novem illæ sunt, ex quibus duæ ipsius $\Delta$ g. ad Frontonem, una Frontonis ad Marcum, altera eiusdem ad Gavium Maximum, ut videtur.

Hunc librum excipit commercium epistolarum inter Frontonem et Marcum, quæ olim secundum temporis rationem in tres partes divisæ erant, ita ut eæ quæ ante huius adoptionem, deinde quæ cum Cæsar vocaretur, postremo quæ cum imperaret scriptæ erant, seorsim haberentur.

2. Unum tantum fragmentum epistolas ad M. Annium Verum servatum est in Cod. quod Maius epist. decimam libri primi ad $L$. Verum fecerat. De eo vide qua scripsimus p. 16.

3. Libri duo epistolarum ad M. Caesarem, seu potius XXIV. ex libris illis epistolæ: minime enim dubium est multo plures intercidisse. Atque inter illas numerantur XIII. M. Aurelii, duæ Frontonis græcæ ad ma-

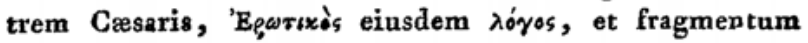
orationis, ut videtur, apud Divum Pium habitæ. Omnes autem vel Frontone consule (a. 143.) vel ante eum annum scriptæ sunt, nec ulla inter eas extat quæ aliqua specie probabili ad illud octodecim annorum spatium referri possit, antequam Marcus de A trelio Cæsare fieret M. Antoninus Ang. Verum incidit in hæc ipsa tempora 4. Enistola ad M. Gresarem de Eloguentia: cuius studium hic repudiaverat. Huius fragmenta quam male in Ed. Mediol. cum reliquiis epistolarum de orationibus confusa sint, demonstravi p. 74. 75 .

5. Epistolarum ad M. Antoninum Aug. libri I. titulo 


\section{XXVIII}

inscripsi paucas illas quæ ex illo libro supersunt, inter quas tres habemus ipsius Augusti: omnes autem scriptre sunt post a. 161. Eas Maium cum epistolis ad Cæsarem commiscuisse monui p. 96 .

6. Epistolae de bello Parthico maxima, ut videtur, pars, scripta a. 162.

7. Epistolae ad M. Antoninum Ang. de Orationibus, quas Imp in Senatu aut habitas aut habendas indicio magistri sui olim commisit. Quæ ex iis supersunt fragmenta trium vel quattuor epistolarum sunt: his, ut dixi, Maius alia omnino diversa intermiscuit, ita ut ex omnibus confusis duos libros effecerit.

8. Epistolae de feriis Alsiensibus quattwor, primis Imperatoris Marci temporibus scriptæ, quorum II. Marci sunt. Hic liber citatur a Charisio.

9. De nepote amisso, tertio fortasse eiusd. Imperatoris anno scriptæ epistolæ, altera consolatoria M. Antonini, altera Frontonis.

Epistolæ quas in hos quattuor libros, 6-9, argumenti causa redegit, quicunque antiquitus opera Frontoniana digessit, parum commode ab aliis separatæ sunt quibuscum coniunctæ sunt: ut ep. de bello Parthico, quæ refertur ad secundam libri ad M. Antoninum: Ep. 1. de orationibus, ad quam profecto respicit quarta Alsiensis.

10. Epistolae ad L. Verum Aug. scriptæ annis $16 \mathrm{r}-166$, quibus insunt quinque Veri, et fragmentum, ut videtur, Panegyrici a Frontone dicti. Plurimæ ex huius libri epistolis initio et fine mutilæ sunt.

11. Libri duo epistolarum ad Amicos, omnes a Frontone scriptæ, quarum itidem permultæ interciderunt. Maior earum pars temporis notis caret satis certis: sunt tamen quas manifesto appareat scriptas esse sub Augustis Fratribus I. 1. 3. 10. 18.: atque idem de pluribus aliis satis liquido perspicere mihi videor : auctoritas vero commendandi, in quo fere omnes versantur, quin Frentoni accesserit ex quo discipuli eius sint imperium adepti, haud dubium. Una est libri secundi sexta, quæ, quum ibi Fronto se filiam desponsasse Victorino dicat, ad Divi 
Pii tempora revocari debere videatur: unde patet non temporum sed hominum, ad quos scripte fuerunt, habitam esse rationem, cum in libros dispertirentur liæ epistolæ.

12. Epistolae graecae quattuor, inter quas una est Appiani Aloxandrini, quas quo tempore scriptæ sint dcfinire nullo modo licet.

13. Principia historiae. Hunc titulum fecit Fronto libro quem gustui misit L. Vero qui illi munus scribendi belli suis auspiciis gesti imposuerat : circa a. 165. Continetille comparationem bellorum Parthicorum Traiani Verique. Et initium et aliae permultæ libri paginæ perierunt.

14. Laudes fumi et pulveris, nugales, ad Marcum iuvenem scriptæ.

15. Laudes neglegentiae, ad idem tempus, ut videtur, referendæ.

16. Arion, ex Herodoto expressa narratio.

Perierunt vero ex Frontonianis, præter ea de quibus neque testimonium extat, neque vestigium ullum:

1. Complures, ut videtur, libri epistolarum ad Marcum Caesarem; quod qui neget, necesse est existimet aut casu quodam non collectas, aut discidium illud de quo indicium facit ep. de Eloquentia, eo usque auctum esse, ut per octodecim prope annos sibi obmutuerint magister et discipulus.

2. Ad MT. Antoninum epistolarum libri post primum omrtes: quum Charisius, qui tamen agnoscat minora illa epistolarum corpora, quintum ex eorum numero landet. Quanquam id fatemur, in tam depravatæ scripturæ auctore parum confidi posse librorum, qui citentur, titulis.

3. Panegyricus T. Pio dictus: cuius meminit Eumenius c. 1/. (vide Fragm. varia p. 270.) Eum non alium fuisse existimo quam orationem quam dixit in Senatu inito consulatu (Lib. I. ad M. Ant. A. 1, 3.) de qua itidem illi sermo est ad matrem Marci (ad M. Ciesarem II. 5.) quin plane eandem ex qua ipse profert quædam (ad M. Cæsarem II. 4.). Quod milii ex eo constare videtur 
quod Plinius (III. 28.) in simili re consulatum sibi iniunxisse offcium dicit gratias agendi Princıpi reip. nomine.

4. Oratio Bithyna seu pro Bithynis, ad Amicos I. ep. 15. 18. Ei post aliquot annos Fronto multa nova addiderat, locum inprimis de acta vita.

5. Oratio pro Demonstruto Petiliano: Ad M. Antonin. Aug. I. 6. Ad Verum Aug. 8. qua oratione compellabatur Asclepiodotus quidam, qui Vero probaretur. Fuit hic Demonstratus homo haud dubie Romanns, erratque omnino Maius qui sibi persuasit eundem esse cun Demostrato illo Atheniensi cui gravissimæ cum Herode intercesserunt inimici:ia. parum tamen sibi constans, quum modo Atticum, modo Cirtensem fingit p. IV. LV.

6. Oratio qua Herodem la serat. ibiden. Quam diversam ab illa pro Demonstrato fuisse mihi certum est: licet aliter censente Maio.

7. Oratio pro Ptolemaeensibus. Clarisius

8. Oratio in Pelopem. Sidonius Apollinaris VIII. ep. 10. M. Fronto cum reliquis orationibus emineret, in Pelopom se sibi praetulit Hunc Pelopem medicum illum esse crediderim satis celebrem, Aelıo Aristidi et Claudio Galeno commemoratum.

9. Adversus Chrtstianos: in qua Christianos nefandorum conviviorum convicio aspersit, ut ait Minucius Felix cap. 9. $\mathbf{3 1}$.

In Editione Mediolanensi nulla extat librorum mentio quos num. 2. 4. 5. recensui : contra fictos errore Editoris quinque libros habet, quos inducendos esse demonstravimus. Sunt autem hi:

Liber secundus epistularum ad Verum Aug.

Liber secundus de oratiunbus ad M. Antoninum.

Pars orations de testamentıs transmarinıs

Pars orationis de hereditate Matıdıæ, cum duabus epistolis.

Pars orationis pro Volumnio Sereno. 


\section{Scripta quae Frontoni falso tribu- untur.}

1. Liber de acie homerica: apud Pseudoælianum qui sub Traiano scripsit, c.1, ibi enim $\Phi$ gorthas pro $\Phi$ górtwrs legi debere, certissimum est.

2. Libri de re rustica, ex quibus extant excerpta in Geoponicis; quæ et sermone produnt diversum a nostro Frontone sequiorisque ætatis auctorem, et de rebus disserunt quotidianis quas illum ignorasse pariter contemptuique habuisse iam nemo dubitabit.

3. Extat in bibliothecis Italicis libellus ex lexicorum. genere, eo insignis quod plurima servavit ex Historiis Sallustianis, atque ex Ciceronis qui interciderunt libris, fragmenta, in quibusdam codicibus inscriptus $M$. Frontonis exempla elocutionun, in aliis vero Arusiani (seu Volusiani) Messi quadriga. Prioris generis librorum auctoritate, seculi XVI. eruditi plerique, qui inde fragmenta hauserunt, Frontoner laudant; Maiusque, de augendo Frontonianorum librorum numero sollicitus, in eorum partes concessit, librumque ex codice Ambrosiano, XVI. seculo scripto, una cum illis edidit. Nullus extat liber cui de suo minus attulerit auctor: neque enim eius proter lemmata verbum ibi invenies, uno fortasse excepto loco v. detraho de te: sed ibi quoque ita barbarus est sermo ut a vetere scriptore profecta esse nequeant. Excerpsit ille ex quattuor scriptoribus (unde quadrigæ nomen inditum) Terentio, Cicerone, Sallustio ot Virgilio, quæ notatu digne viderentur elocutiones, ita ut ipsas litterarum ordine lemmatis loco poneret, deinde verba subiungeret scriptoris apud quenı invenisset, tali modo: Abundans illius rei. Virg. Ecl. Nivei quan lactis abundans. - Urbs et nomini plurali subiunctum. Virg. Aen. X. Quique urbom liquere Cosas. Cicero de Siynis. Urbem Syracusas maximam esse graecarum. Iam vero, postquam Frontonis de romani sermonis auctoribus iudicium innotuit, qui de Virgilio et Terentio taceat, Ciceronem antiquio- 


\section{XXXII}

bus certe non præferat, quis illum credat, si huius generis libro consarcinando operam dicasset, non Catonem potius illum sunm, Enniumque oum C. Crispo adhibiturum fuisse? Itaque librum qui tam gravi indicio Frontoni abiudicetur, et ita tennis, et collectis inde a Carrione, Patricio et Sigonio fragmentis iam nulliu fructus sit (1), qui præterea a parte testium alii auctori

(x) Etiam in hoc libro recensendo eximia illa sua diligentia usus Maius omnia quae in eo extant loca in editis quaesivit libris; quaedam frustra, quae ideo in unum collecta proponere haud inutile visum est, quia incognita hucusque fragmeuta illis inesse videntur. Quod de illis quae ex oratione in Pisonem afferuntur certo affirmare ausim: quae vero ex orationibus integre servatis Maio falso citari videntur, utrum aut in his aut aliis locis inveniantur quaerendi otium defuit : indicavit autem amicissimus Boeckhius quae ex oratione de Signis landantur: temeritati et mendacio conscius ibi legi c. 56. recte scripta t e.m,meo conscios: illa : dum armis exercetur, ibidem c. 25 : denique in eadem oratione, eiusque cap. 5o. extare illa quae ex oratione de suppliciis laudantur: neque tam fugitivi illi a dominis quam tu ab iure et a legibus neque tam illi hostes hominitus quam tu diis immortalibus. Atque id iam bona fide aftirmare possum, ita exhaustum esse libellum ut nullam amplius praestare possit utilitatem.

\section{Ex Cicerone.}

Incerto libro. Neque adsidere Gabinium aut alloqui in curia quisquam audebat.

De republica. A quo isti avocant ibid. Ut de via decedendum sit.

ibid. Sermonem in mulram noctem produximus.

In Pisonem. Homini levi et subito filium (l. fliam) collocavit. (Sermo est de avo I'isonis materno, ut apud Asconium pro paterno scribenduin esse p. I56. Ed. Lugd. x644. moneo.J

ibid. Dedecus quemadmodum ingressus.

ibid. Maiorem sibi Insuber ille avus adoptavit.

In Siciliensi. Tecum tuum conclusisset. ibid. $\quad$ Cum eo coniuncta est

In Frumentaric. Fundus erat colono locatus.

De Suppliciis. Quae tu de Kep. detraxeris. ibid. Ut exeunt e Sicilia praedones.

Pro C'luentio. Sexcentis millibus numum se iudicium corrupturum esse dixerat.

ibid. Diffidentem rebns suis.

ibid. Quotiescumque quicquam eiusmodi monstri poterat in animum inducere.

ibid. Ingenio et virtute par his quos ante memoravi.

Pro Flacco. Quam benivolum hunc P. R., quam fidelem putatis? (Horum simillime Phil. XI, 15. extare recte monuit AIaius.) 
diserte tribuatur, non recepimus, quum præsertim moli sumptuique editionis nostræ parci voluerimus.

Raphael Volaterranus inter libros qui a. 1494 in Bobiensı bibliotheca inventi fuerint, Cornelii Frontonis elegantias lacinas recenset: quæ si fuerunt exempla de quibus hic sermo est, vetusti quidem Codicis auctoritatem habet qui illa Frontoni rindicat; ea tamen quam intirma sit unter omnes qui sapiunt convenit.

\section{Editio Mediolanensis.}

Prodierunt Frontonianæ reliquiæ Mediolani 1815, duobus voluminibus, in splendida charta, et typis sane decoris, imaginibus Augustorum, ad quos epistolas scripsit Fronto, ornatæ, et specimine scripture instructæ. Titulum editio præfert hunc:

III. Cornelii Frontonis opera inedita, cum epistulis itom ineditis Antonini Pii, M. Aurelii, $L V E$ ri ot Appiani, nec non aliorum veterum fraymentis. Invenit, et commentario praevio notisque illustravit Angelus hIaius. Pars prior. Pars altera, cui adduntur seu edita seu cognita eiusdem Frontonis opera. Modiolani, regits typis.

Ceterum præter oxempla illa elocutionum nihil ibi extat quo nostra careat editio (1).

De domo. Reip. vicem Iugeo. (Mtale Mraius: de Domo et Rep Vicem l.)

Pro Sextio. Negotii gerentes. (Malus conferre iubet or. pro Quintio c. rg.)

Ex Sallastio.

Iugurtha. Imperii prolatand percupidus habebatur, cetera egregius.

(I) Erenm aliena plane ab hoc opere sunt p. $430.45 x$, variae lectunes adnudum paucae exCod. Ambrosians ad illa quibus lacunam in l'uncis Appian supplevit $\mathrm{Cl}$. Schwe, haenserus zum p. $548-550$ Prologus tlemantii Martyru do V. et B. so. eal, et specimen glossarum Placid. 


\section{Codex Frontonis Ambrosianus.}

Delituerant hæ reliquiæ in codice ex genere rescriptorum, de quo pauca admodum nos docet inventor Maius. De indole illins quæ is diversis locis tradit, collecta fere hæc sunt. Bxtare in bibliotheca Ambrosiana, ex Bobiensi cœnobio allatum (1) codicem, forms maxima, quam in folio dicunt (2); quo contineri latinam interpretationem Actorum Concilii Chalcedonensis, antiqua manu scriptam, quæ ad VIII. seculum referenda esse videatur (3). Ad eum librum conficiendum adhibitas esse membranas plurium veterum codicum, qui, credo, extabant in cœnobii bibliotheca (4): Frontonis, Interpretis antiqui ad octo orationes Ciceronis (5), Symmachi orationum (6), et panegyrici Pliniani (7): aliorum fortasse preterea, de quibus nullam mentionem Maius fecit. Binas unamquamque Frontonis paginam columnas habere inde colligo, quod id de Interprote Ciceronis tradit Maius ( $(\mathrm{S})$, idemque quacumque de huius codicis scriptura narravit, de illo pariter affrmat (9). Atque ipsum æri incisum

(r) Maius in praevia diss, ad Interpr. Cic. p. IV.

(2) Ingentes paginas dicit Maius p. 336. Ed. Med. et p. 592. lacumam esse integrae paene paginae Codicis in $f^{\circ}$. (sic)

(5) Mains ad Interpr. p. $r$. Ego, quantum ex specimine indicare licet, recentiorem esse arbitror.

(4) Nescio an quisquam animadverterit fragmentum quod Muratorius edidit catalogi Bubiensis bibliothecie inter libros yui illic habiti sint memorare Septimit Sereni upusculat ruralia: quae cum pariter resscribendo destructa esse consentaneum sit, viros doclós Mediolanenses, ipsum ante alios praestantissisum Mainm, summopere rogamus, ut ea in Ambrosiana investigare laborent.

(5) Miaius 1. I. Hic Ciceronianus interpres magni sane pretii est, et docius, non tamen est Asconius, sed idem qui in Verrinas scripsit, quarti seculi homo. Nam is quoque commentarius non recte Asconio tribuitur, atqne mihi dubium non est, in Poggianis Ascouii aposraphis hunc non extitisse, sed aliunde editum esse.

(6) Maitis praef. ad Symm. p. XIII,

(7) Ma:us ad Symm. ed n. p.62.

(8) Biss. ad Interpr. Cic. p. V.

(9) Comm. praev, ad Front, p. CL, 
specimen, quanquam unius tantum columnæ, hoc confirmare videtur (1). Quo specimine diligenter inspecto fore ut paucissini illi qui perfectam palæographix coguitionem habent, accurate definiant quo tempore exaratus sit Frontonianus codex, optandum magis est quam sperandum, quoniam fallax admodum de ea re iudicium est. Mihi ut tantum haud tribuerim, ite tamen dicam me in libris qui vix ante sæc. VII. initium scripti esse possint, idem plane litterarum scribendique genus vidisse, quin pulchriores etiam ductus quam quos Frontonianze membranæe exlibent: quos quum Maius ad quartum seculum referat (2), audacius mihi pronuntiare videtur; multo antiquiorem esse Tullianarum orationum quas in Sedulii codice invenit scripturam, recte dictum arbitror. Frontoniani quidem codicis soríptura Pandectarum librum Florentinum in permultis refert, atque etiam iuneturam litterarum $\mathbf{N}$ et $\mathrm{T}$. In aliis tamen differunt, neque apparet in Frontoniano codice $S$ illud ex cursiva petitum scriptura : et in universum elegantior multo minusque festinatus est Frontonianus codex. Vocabula continuantur: id vero singulare est, quod , membra oratio"nis distinguuntur puncto crasso recurvo superiori vo,cabuli parti adscripto " quam distinctionis notam agnoscit Diomedes, ut bene docet Maius. In Gracis nulla distinctio deprehenditur (3). Titulus librorum in summis paginis scribitur minutis litteris: grandibus initio et fine librorum: neutro loco adduntur sollemnia illa incipit et explicit (4). Numeri nota in summa pagina non nisi secundo libro additur (5). Epistolarum clausula certe in specimine quod æri incisum est, angustioribus versibus continetur: quod ideo observo, quod id in ipsarum quæ mittebantur epistolarum forma usitatum

(x) Omisituus illud, quum sufficiat talia semel extare.

(2) 1. l. Counm. praer.

(3) Ibid.

(4) V. Specimen Codicis, columna secunda, tum ed. n. p. ro4. 155.

(5) P. 104. 


\section{XXXVr}

fuisse videri potost (1). Emendatus codex erat collatis exemplaribus, a quodam, qui suum se nomen in codicis initio, oum quo simul periit, adscripsisse testatur formula in fine posita Legi emendavi qui supra (2), qua septies occurrit (3); idem in margine quæ sibi notatu digna viderentur, indicnvit lemmatis: scholia certo dici son possunt: littera in illis usus oursiva, qua quum sec. V. ut ex chartis apparet, in usum recepta sit, cove indo cum Maio colligas eius laborem multo recentiorem esse cetera codicis seriptura. Fomanum fuisse librarium ex gracis qua scripait apparet (4): minime tamen ita ignae rum sermonis græci ut Maio placet (5); errores enim qni in illis occurrunt fere omnes ex male lecto, non ex male scripto codice nati sunt.

Orthographiam ut Maius expressit, ita servavimus, licet pleraque in quibus a recepta differt ex vitiato in barbariem prolapgi æevi usu nata sint. Ea in plurimis cum Pandectis Floyentinis consonat: uterque enim liber exhibet inter alia epissrula, neglegentia, thensaurus: et in utroque pariter $B$ ponitur pro $V$, et $\nabla$ ubi $B$ esse debet.

„Folia in codice cuncta, incredibili quadam pertur,batione commixta, materizeque ordinem funditus subla"tum esse, ea se in ordinem disposuisse: " ad Cicerunis interpretem docet Maius (6): quod hoo loco, ad confirmanda ea qux in epistola ad Iægerum scripsi, addere quam omittere satins duce, postquam illo Maii liber demum his ipsis diebrss ad me allatus est.

Quod multi oonquesti sunt rescribendorum librorum more, quam ecclesiasticis scriptis auctores veteres cedere cogerentur, permultos periisse optimi ævi libros, internpestivum fuerit repetere: quin fatendum est, potuisso ita servari quæ a rudibus illorum seoulorum ingeniis, pisi sibi utilia tecissent, aut neglecta aut abiecta essent:

(r) Spec, Godi-eol. $x$.

(2) Connu. praev p. CIL. CIrI.

(5) P 95. II2. 13I. 152. 208. 253. 264. Semel rero recogaovi p. ro4.,

(4.) P. 235 . adn. r. ed, n.

(5) P. 40. adn. 4. ed. n,

(6) P. VI. 
que inter tot bella, tot popnlationes et ruinas, plurimis libris pereundi causa extitit. Sed industria diligentinque nostra dirigenda est ad inveniendos qui plurimis locis procul dubio adhuc delitescunt litterarum thesauros; atque ut evanidi ductus reviviscant, ars chemica in subsidium vacanda est. Ceterum, ut hoo addam, qua in aliquammultis libris Latinis, (nam apud Græcos divites, et ab antiquis studiis non alienos rarissime factum est, quod paupertas et barbaries Italis suadebat), quæ in Latinis, inquam, libris nonnullis desunt folia, quorum numerum nonnunquam codices indicant, ea et ipsa ad rescribendi usum esse ablata videntur. Et mihi quidem persuasum est, etiam Petronii qua supersunt evasisse cladem, ex eadem causa illatam, cum pars foliorum, ut alium textum reciperet, ex codice evulsa esset, reliqua manerent: quorum tamen plurima folia in eundem usum, abraso ablutoque atramento, proparata essent: unde factur est, ut in integris paginis pauci versus, neque inter se coniuncti, legi potuerint $a b$ eo, qui, littexarum studio renascente, in alium transcriberet codicem.

\section{Alii Frontones.}

Frontonum brevissimam subiiciamus his mentionem. Multi eo nomine sub Imperatoribus fuernut, nulla inter so cognatione iuncti. Octavius Fronto, pretorius, Tacit. Annal. II. 33. Vibins Fronto, magister equitum, ibid. II. 68. Inlius Fronto, trib. vigilum, Tacit. Histor. I. 20. II. 26. Catius Fronto fuit orator, Plinii minoris aqualis, qui pro Basso et pro Vareno dixit, Plin. IV. 9. V1. 13. Inuc eundem esso autumo cuius platani et marmora Invenali memorantur, Horatiana domus dominum. Claudius Fronto Asiarcha sub Antonino Pia et L. Vero in numis XIJI. Asia civitatum: de quo vido Maium in Comm. previo p XXIV. seqq.: fortasse non diversus a Frontone, cuius mortui admodum honorifice meminit Ael Aristides in Genethliaco Apellæ T. I.p.125. B. ed. Cant. L. Caecilius Fronto Smyrnensis a'yurogirns, fortasse sub T. Antonino Pio, apud Spon. Miscell. X, 93. 


\section{XXXVIII}

p. 353. Dapirius Fronto IC. qui laudatur a Callistrato et Marciano in Pandectis. Fronto Emisenus, Longini avunculus, qui sub Severo Imp. Athenis rhetoricen professus est, multasque orationes scripsit: v. Suid. s. v. Фgérray. Fronto Medious in inscriptione Græca Catanæe ap. Castell. Princ. Turr. Mut. Inscr. Sic. XIV, 48. p. 181. ed. sec. ubi nunc male legitur $\Phi 10 N T \Omega N$. Tum M. Aufidius Fronto, Cornelii nostri ex filia nepos, de quo v. supra p. XXV. - eiusque filius infans M. Aufidius Fronto: Q. Aufidius Fronto et $C$ n. Domitius Fronto ap. Gruterum CMXXXIII. 6. M., Marius Fronto ap. eundem DCXCV. 9. Fronto libertinus, Cod. Iust. X, 88. 2. Fronto Arvernus, avus Apri, qui familiaris Sidosii fuit, IV. ep. s1. Fronto Caristanus, et Valerius Fronto, apud Apsyrtum in Hippiatricis. Fronto II oir Enonæ in inscript: quam laudat Maius, comm.præv.p. VII. Fuerunt etian, quos itidem suppeditat Maius, Fronto monachus, et duo Frontones martyres, quorum alter in Africa, alter Crsaraugustæ passus est. M. vero Cornelium Frontonem III. Cos. Traiani Imp. collegam a. 100, qui Sex. Iulius

Frantinus esse debeat, Norisius fastis expulit. 